\title{
A Contemporary Overview of the History and Applications of Artificial Life
}

\author{
Jamaluddin Mir ${ }^{1}$, Majid Mehmood ${ }^{2}$, Malik Touqir Anwar ${ }^{1}$, M. Yaqoob Wani ${ }^{1}$ \\ ${ }^{1}$ SIT Department, the University of Lahore, Islamabad, Pakistan \\ ${ }^{2}$ Computer Science Department, University of Gujrat, Sialkot, Pakistan
}

Email address:

mir.jamal@uolisb.edu.pk (J. Mir), majid.mehmood@uogsialkot.edu.pk (M. Mehmood), malik.touqir.anwar910@gmail.com (M. T. Anwar), yaqoobwani@gmail.com (M. Y. Wani)

\section{To cite this article:}

Jamaluddin Mir, Majid Mehmood, Malik Touqir Anwar, M. Yaqoob Wani. A Contemporary Overview of the History and Applications of Artificial Life. Automation, Control and Intelligent Systems. Vol. 3, No. 1, 2015, pp. 6-10. doi: 10.11648/j.acis.20150301.12

\begin{abstract}
The study of man-made systems which demonstrate certain behaviors that are found to be distinctive characters of natural living systems, found in nature, is called Artificial Life. Artificial life supplements the classic biological science which is focused on the investigation of living organisms by trying to produce life-like characteristics in computer and other such machines. Artificial life is focused on developing an understanding of the fundamental doctrines of life by either creating lifelike characteristics in simulations created by computers or by actual physical implementations. Though, the aim of artificial life is concentrated towards both the future and origin of biology, yet the complexity of the subject area requires involvement of other fields of science. The practical as well as the scientific impact of the field of artificial life are equally far reaching.
\end{abstract}

Keywords: Artificial Life, Artificial Intelligence, Evolution, Cybernetics, Tierra, Self-Replicating Systems

\section{Introduction}

Since 1980s, the field of artificial life has examined various living systems by an artificial and synthetic approach, with the aim of developing a better understanding of life by means of software. Researchers are of the opinion that the first artificial life model was developed in 1951 by von Neumann [1]. The concept of automata of was described on a cellular level and a self-replicating system was proposed with the intention of developing a universal formal computation system that will be prone to unrestricted evolution [2, 3]. Self-replicating loops were proposed in 1984 [4] which were a lot simpler.

At the same time, the researchers from the field of cybernetics were focusing on the control and communication aspects in these systems $[5,6]$. The discipline of cybernetics aims to describe the phenomena in regard to their respective functionalities instead of the substrate. It was proposed by Langton [4] that life demands to be studied as a property of form rather than matter. This is in close relation to the concepts followed by cybernetics. So it can be safely said that ALife is closely related to the field of cybernetics. Furthermore, the fundamental theories of homeostasis [7] and autopoiesis [8] are derived from the field of cybernetics.
Apart from cybernetics, Alife and artificial (AI) are also related where ALife is more focused on systems which can simulate the nature and its laws and AI is aimed at simulating the human intelligence.Another major difference is that of the different modeling strategies used by ALife and AI.

\section{ALife and AI}

Although ALife and Artificial Intelligence (AI) are quite different from each other, yet both the fields of computer science are somewhat connected by means of ALife's roots in machine learning. The fields of ALife and AI depict a certain amount of similarity as both these fields of computer science rely on similar methodologies, erupting from natural phenomenon. However, there are remarkable differences between AI and ALife. Majority of the classical methods of AI are serial systems that best defined in a topdown manner, comprising of very complex controllers that are centralized and make decisions on the basis of global knowledge of the system. The decisions made by the centralized controller are very much capable of having an impact on any part of the system. Whereas, in contrast to this approach, numerous living systems existing in nature demonstrate highly complex and self-governing behavior which is parallel in nature, depicting the behavior of a 
distributed network, comprised of low-level agents. The decisions made by each agent are made on the basis of their local environment and local knowledge. As can be seen, this approach is in contrast to the centralized approach of the AI models where on centralized controller makes decisions on the basis of global knowledge. The ALife models are defined in a bottom-up manner which is characteristic of simple agents that are parallel in nature and act in a local manner. These types of models are called agent-based models. The overall behavior of the system emerges as a result of the interactions among the agents.

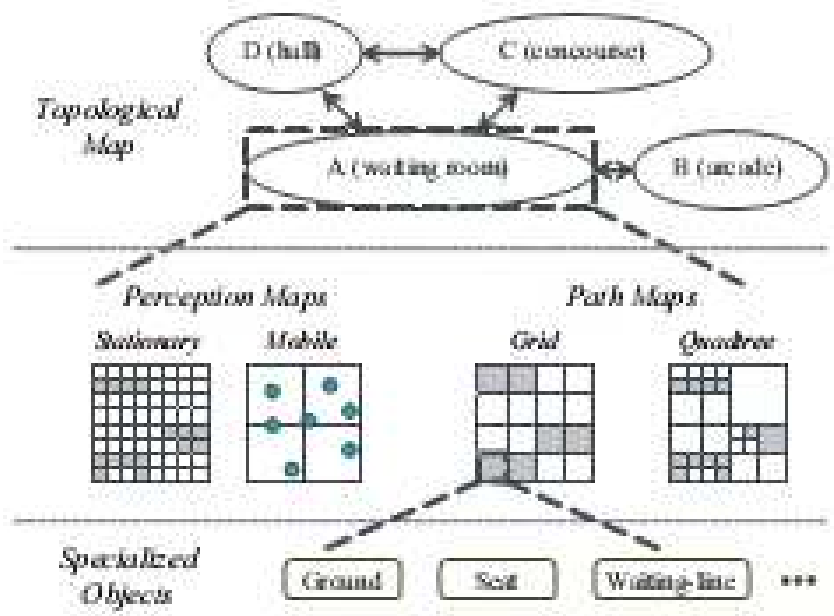

Figure 1. Hierarchical model for ALife.

\section{Digital Evolution}

Researchers believe that the most efficient way to study the issues about evolving systems is by their implementation in the software. This approach has been central trend in the field of ALife. The earliest significant such implementation in the digital world was Tierra [11]. Simply put, Tierra was a population of computer program that was self-replicating in nature and resided in the computer memory. The genotype comprised of machine code, with each Tierran creature an instance of some genotype. The simulation of the program started with the self-replication of a single ancestor that was placed in the computer memory. The process of self-replication by the ancestor and its subsequent child continues uptil the point when the computer memory is full of such creatures, sharing the same genotype of their ancestor. Among the overall population, older creatures are eliminated so as to create space for new progenies. The population evolves by means of natural selection in case of any errors or mutation as it is said. If a certain error or mutation allows the program to replicate quickly, that particular mutated genotype will tend to spread among the population. As a result, over time, the population becomes extraordinarily diverse. Subsequently, millions of CPU cycles later, the Tierran population now contains several different types of creatures that demonstrate various different relationships in their ecology.

\section{Categories of ALife}

ALife has been categorized into three extensive but interrelated branches that relate to three different respective methodologies. The majority of the ALife research is in the area of Soft ALife which is aimed at creating simulations depicting life-like behavior. Hard ALife is aimed at developing hardwareimplementations. Wet ALife is aimed at producing living systems from certain bio-chemical materials [9]. In broader sense, therefore, it can be said that ALife is the synthesis and simulation of living systems which is aimed at developing a better understanding of life and life forms by simulations.

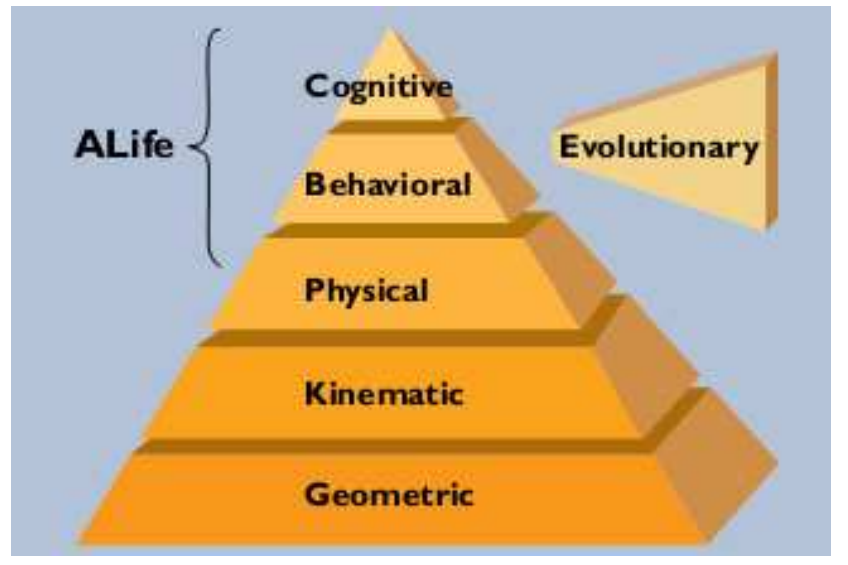

Figure 2. Modeling for Artificial Life.

\subsection{Autonomy}

Since its inception, ALife has been closely related to the theories of biological autonomy and autopoiesis [10]. Autopoiesis describes the constrained grid of processes which self-maintain the organization in such a manner that it makes them easier to be identified in a chemical substance [11].

\subsection{Self-Organization}

Self-organization is the ability of systems where local interaction can result in global behaviors for example swarm of bees, flock of birds or traffic pattern $[12,13,14$, 15]. The traditional examples of self-organization in ALife are snowflakes [16] and boids [17] which use pattern formation and cooperative motion [18]. Some researchers argue that the characteristic of self-reflection is a special case of the concept of self-organization because in the process of replication, the object under consideration can conserve as well as duplicate its organization on its own. Self-maintenance is also another exceptional case of selforganization that is more in close relation to homeostasis [19]. Researchers have developed very sophisticated robots which are based on the swarm intelligence. Such feature of self-organization has been a motivation for computational intelligence. 


\subsection{Adaptation}

Any change in an agent or within a system in reaction to the environment which will consequently help that particular system or agent to achieve their goal is called adaptation [15]. Researchers consider adaptation to be the fundamental characteristic of all living systems that is extremely important for autonomy and the very survival of the system or agent. In contrast to AI which is more concerned with predicting and controlling, ALife is more focusing in developing the capability of adaptability in AI [20]. However it must be noted that predictability is as much important as is adaptability, both in natural systems as well as in artificial systems.

\subsection{Evolution}

Evolution has been one of the key concepts explored by computer sciences with prime focus on evolutionary algorithms like genetic algorithm [21] and computational intelligence [22]. All such evolutionary algorithms are focused on finding the best possible and optimum solution out of an infinitesimally large search space.

\section{Applications of ALife}

\subsection{Artificial Societies}

Researchers have described the societies on the basis of relationships between the individuals belonging to the same species. Investigation of all likelihoods of social interactions which used to be very difficult to explore in complex systems has now become possible by the help of computational modeling [23]. The societal modeling concepts have paved the way for developingALife games likeCreatures and TheSims [24].

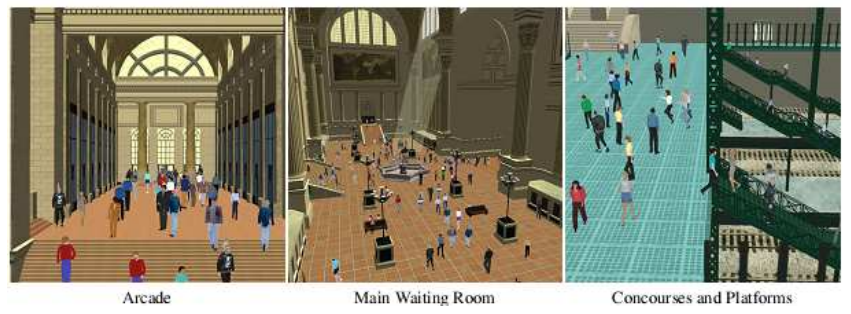

Figure 3. A large scale Artificial Society created by ALife models.

\subsection{Computational Biology}

Neural networks as well as evolutionary algorithms have found the very basis of modern computations and have proved that concepts derived from biology can be used as the central concepts in other sciences. Neural networks, derived from biology, have been used to develop distributed computation models. Many of these have been used by ALife in different stages and models. The biological immune systems have served as motivation and central logic of numerous new advancements in the field of computer security, as well as in the field of optimization [24].

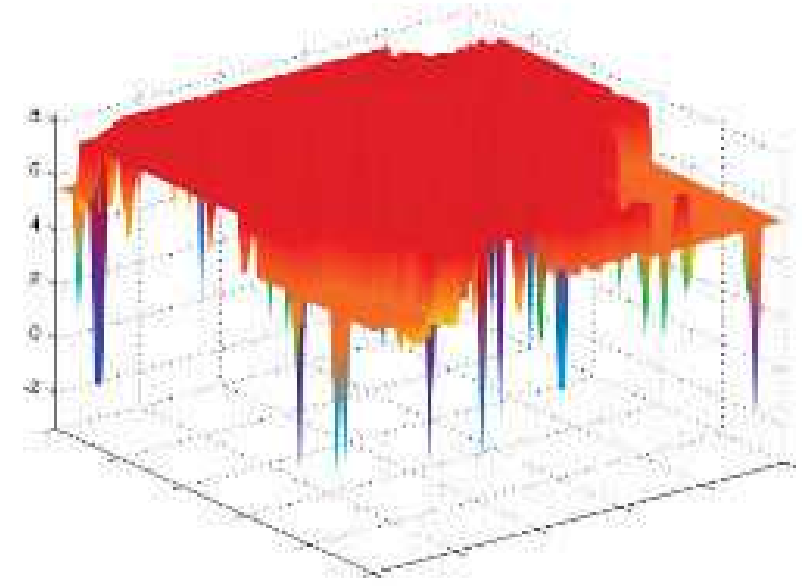

Figure 4. Avida; an ALife software program in which the bits of code depict actual organisms.

\section{The Future}

Researchers believe that an improved understanding of life will help in enabling us to make intelligent decisions in regard to environmental resources, social life, development of cities, commercial development of biotechnology and many other areas of applied sciences.

The sophisticated nature of the living systems has been the limiting factor when it comes to the scalability of artificial systems that are to be designed. Such concepts can be applied to electric circuits as the relations of the components can be regulated and optimized. The concepts and perceptions of ALife are slowly and gradually infiltrating biological sciences the same was as complex computational modeling is making its way into all fields of biological sciences. If the current trend continues in the field of ALife, soon we would have to revise ourconcepts about real and artificial.

Although artificial life (ALife) is related to artificial intelligence (AI) yet there are very critical differences between the two subject areas, mainly among the modeling strategies used by AI and ALife. Majority of the classic AI models are top-down identified serial systems which use a very sophisticated and centralized controller which is responsible of making decisions on the basis of all the states. The decisions of the controller are capable of affecting any or all thesystem aspects of the whole system. In contrast, as can be observed, majority of the natural systems are parallel or non-linear, having inter-relations based on agents that interact with each other. The decisions of the agents are based on the information available specifically to that particular agent and they are so designed that the decisions of the agents have an impact only on the location of that particular agent. The models followed in ALife are specified by following a bottom-top non-linear systems of agents which interact in their local capacity. As a result of these several local interactions between agents, a global change can be observed.

The synthetic approach of ALife has numerous advantages. The fact that a theory related to life can be expressed by computer code renders it an extremely high level of accuracy 
and precision. Computer generated models have been observed to simplify the extent of conceptualization that is found to be a prerequisite for constructing outstanding models of natural phenomena.

An important part of the research conducted in the field of Alife is mainly concerned with implementing the research in real life situation, instead of merely carrying out computer simulations. As a result, such products and systems can be engineered which exhibit life-like characteristics. However, a significant part of the research conducted in this field is purely theoretical and aimed to develop a better understanding of life by understanding the very essence of life. The problems that can be solved by ALife can include developing electronic hardware tobio-inspired robotics by making use of evolutionary algorithms which simulate nature inspired processes like the concept of swarm intelligence of bees and flocks of birds by actual swarm of robots.

\subsection{Health/Medicine}

The growing complexity of the current health-care system has made it ideal for applications from ALife [25]. The ALife applications demand a thorough understanding of very nature of the health-care system which encompasses the understanding of the behavior of the system, responding to the changes in the system, dealing with the monetary issues and, in future, making the whole system adaptable to nonlinearity of the real world issues.

\subsection{Transport}

In most of the metropolitan cities all around the world, the traffic and transportation system poses a large scale complexity and non-linearity. Models and simulations from ALife can be applied to the traffic movement as well as for developing infrastructure network [26]. The number of engineering applications in ALife are only bound to increase in the near future.

\subsection{Artificial Societies and Artificial Economies}

The ALife models have been vastly applied into what is now described as artificial societies and artificial economies. The agent-based modeling which uses the bottom-up effect among the social systems has been used to generate models which can be simulated in artificial environments. If the bottom-up effect methods of ALife are applied to the markets, the artificial societies evolve into artificial economy. The economic systems are highly complex entities which face several non-linear components and variables. In this regard, the ALife models have a natural ability to deal with all these non-linearities that deal with the non-equilibrium phenomenon.

\section{Conclusion}

A majority of the real world problems were solved by mathematical programming methods. However, it must be noted that a large portion of the real world problems which were traditionally solved by the optimization methods and mathematical programming techniques were somewhat smaller in scale. Many of the engineering problems in the real world are combinatorial, consequently posing large dimensionality as it is very difficult to modularize all such problems. Since its inception, ALife is slowly integrating into almost all fields of sciences. Specially in all such environments where a human controller is required to make complex decisions based upon several different variables, the ALife based models can simplify the decision making process, thereby making things easier.

\section{References}

[1] von Neumann, J. (1951). "The general and logical theory of automata," in Cerebral Mechanisms in Behavior-The Hixon Symposium, 1948 (Pasadena CA: Wiley), 1-41.

[2] von Neumann, J. (1966). The Theory of Self-Reproducing Automata. Champaign, IL: University of Illinois Press.

[3] Mange, D., Stauffer, A., Peparola, L., and Tempesti, G. (2004) "A macroscopic view of self-replication," in Proceedings of the IEEE, Number 12. IEEE. 1929-1945.

[4] Langton, C. G. (1984). Self-reproduction in cellular automata. Physica D 10, 135-144. doi:10.1016/0167-2789(84)90256-2.

[5] Wiener, N. (1948). Cybernetics: Or, Control and Communication in the Animal and the Machine. New York, NY: Wiley and Sons.

[6] Gershenson, C., Csermely, P., Erdi, P., Knyazeva, H., and Laszlo, A. (2014). The past, present and future of cybernetics and systems research. Systema. 1, 4-13.

[7] Ashby, W. R. (1947a). The nervous system as physical machine: with special reference to the origin of adaptive behavior.Mind 56, 44-59. doi:10.1093/mind/LVI.221.44

[8] Varela, F. J., Maturana, H. R., and Uribe, R. (1974). Autopoiesis: the organization of living systems, its characterization and a model. Biosystems 5, 187-196. doi:10.1016/0303-2647(74)90031-8

[9] Rasmussen, S., Chen, L., Nilsson, M., and Abe, S. (2003). Bridging nonliving and living matter. Artif. Life 9, 269-316. doi:10.1162/106454603322392479

[10] Bourgine, P., and Varela, F. J. (1992). "Introduction: towards a practice of autonomous systems," in Toward a Practice of Autonomous Systems: Proceedings of the First European Conference on Artificial Life, eds F. J. Varela and P. Bourgine (Cambridge, MA: MIT Press), xi-xvii.

[11] Maturana, H., and Varela, F. (1980). Autopoiesis and Cognition: The Realization of Living. Dordrecht: Reidel Publishing Company.

[12] Haken, H. (1981). "Synergetics and the problem of selforganization," in Self-Organizing Systems: An Interdisciplinary Approach, eds G. Roth and H. Schwegler (New York, NY: Campus Verlag), 9-13.

[13] Camazine, S., Deneubourg, J.-L., Franks, N. R., Sneyd, J., Theraulaz, G., and Bonabeau, E. (2003). Self-Organization in Biological Systems. Princeton, NJ: Princeton University Press. 
[14] Gershenson, C., and Heylighen, F. (2003). "When can we call a system self-organizing?," in Advances in Artificial Life, 7th European Conference, ECAL 2003 LNAI 2801, eds W. Banzhaf, T. Christaller, P. Dittrich, J. T. Kim, and J. Ziegler (Berlin: Springer), 606-614.

[15] Gershenson, C. (2007). Design and Control of SelfOrganizing Systems. Mexico City: CopItArxives.

[16] Packard, N. (1986). "Lattice models for solidification and aggregation," in Theory and Application of Cellular Automata, ed. S. Wolfram (Tokyo: World Scientific, Institute for Advanced Study Preprint), 305-310.

[17] Reynolds, C. W. (1987). Flocks, herds, and schools: a distributed behavioral model. Comput. Graph. 21, 25-34. doi: $10.1145 / 37402.37406$

[18] Vicsek, T., and Zafeiris, A. (2012). Collective motion. Phys. Rep. 517, 71-140. doi:10.1016/j.physrep.2012.03.004

[19] Williams, H. T. P. (2006). Homeostatic Adaptive Networks. $\mathrm{PhD}$ thesis. Leeds, UK: University of Leeds.

[20] Brooks, R. A. (1991). Intelligence without representation. Artif. Intell. 47, 139-160. doi:10.1016/0004-3702(91)90053M.
[21] Holland, J. H. (1975). Adaptation in Natural and Artificial Systems. Cambridge, MA: The University of Michigan Press.

[22] Gilbert, N., and Conte, R. (eds) (1995). Artificial Societies: The Computer Simulation of Social Life. Bristol, PA: Taylor \& Francis, Inc.

[23] Grand, S. (2001). Creation: Life and How to Make it. Cambridge, MA: Phoenix.

[24] Burke, E. K., Kendall, G., Aickelin, U., Dasgupta, D., and Gu, F. (2014). "Artificial immune systems," in Search Methodologies, eds E. K. Burke and G. Kendall (Springer), 187-211.

[25] Plsek, P. E., \&Greenhalgh, T. (2001). The challenge of complexity in health care. Bmj, 323(7313), 625-628.

[26] Bauer, D. C., Cannady, J., \& Garcia, R. C. (2001). Detecting anomalous behavior: optimization of network traffic parameters via an evolution strategy. In SoutheastCon 2001. Proceedings. IEEE (pp. 34-39). IEEE. 